PNNL-12194

Unlimited

\title{
PNNL Review of Proposed Relevant Radionuclide List
}

Dr. Harry S. Miley

Dr. Richard J. Arthur

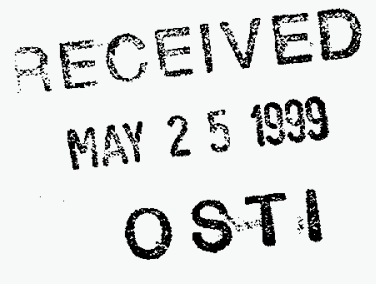

May 1999

Prepared for the U.S. Department of Energy under Contract DE-AC06-76RLO 1830 


\title{
DISCLAIMER
}

This report was prepared as an account of work sponsored by an agency of the United States Government. Neither the United States Government nor any agency thereof, nor Battelle Memorial Institute, nor any of their employees, makes any warranty, express or implied, or assumes any legal liability or responsibility for the accuracy, completeness, or usefulness of any information, apparatus, product, or process disclosed, or represents that its use would not infringe privately owned rights. Reference herein to any specific commercial product, process, or service by trade name, trademark, manufacturer, or otherwise does not necessarily constitute or imply its endorsement, recommendation, or favoring by the United States Government or any agency thereof, or Battelle Memorial Institute. The views and opinions of authors expressed herein do not necessarily state or reflect those of the United States Government or any agency thereof.

\author{
PACIFIC NORTHWEST NATIONAL LABORATORY \\ operated by \\ BATTELLE \\ for the \\ UNITED STATES DEPARTMENT OF ENERGY \\ under Contract DE-AC06-76RLO 1830
}

Printed in the United States of America

Available to DOE and DOE contractors from the

Office of Scientific and Technical Information, P.O. Box 62, Oak Ridge, TN 37831; prices available from (615) $576-8401$.

Available to the public from the National Technical Information Service, U.S. Department of Commerce, 5285 Port Royal Rd., Springfield, VA 22161

This document was printed on recycled paper.

$$
\text { (9/97) }
$$




\section{DISCLAIMER}

Portions of this document may be illegible in electronic image products. Images are produced from the best available original document. 


\title{
PNNL Review of Proposed Relevant Radionuclide List
}

\author{
Dr. Harry Miley and Dr. Richard Arthur \\ Pacific Northwest National Laboratory \\ Richland, WA 99352
}

\section{Introduction}

A list of fission products and activation products has been proposed for possible adoption as an official table of relevant isotopes for CTBT use. It is our understanding that the purpose of this list is to discriminate Level 4 spectra from Level 5 spectra in the decision logic diagram. The current understanding is that a single short-lived, relevant isotope that is atypical for a station would cause a spectrum to be marked as Level 4 . A second relevant isotope would cause a spectrum to be marked as Level 5 , which would perhaps require a sample to undergo additional laboratory confirmation measurements.

The list consists of a very comprehensive set of fission products and activation products. We have examined the list for accuracy and have also flagged potential problems with members of the list. In our opinion, several of these isotopes have serious problems and many have no practical chance of ever being the first or second detected isotopes. We are not arguing whether or not these isotopes might be seen in a large atmospheric test. On the other hand, there may be no harm associated with having a long list.

The issue of activation products is different. Some activation products are indicative of the soil or rock composition in the vicinity of an explosion. Others may only be dependent on materials in the weapon or in the support structures. We don't think that a great deal of analysis of these isotopes by the CTBTO should be encouraged. In any case, if particulate activation products are in the atmosphere, fission products should be even more prevalent, thus removing the need for an activation list component.

In order to inject some reality into the discussion, we point out that for 11 historical low-yield Chinese atmospheric tests detected at PNNL $(\sim 20 \mathrm{kt}$, range $10,000 \mathrm{~km}$, travel time $\sim 12$ days), a RASA would have collected an average of $6 \times 10^{9}$ fission atoms on a filter. Since the detection threshold for the RASA (and other CTBT RN aerosol systems) is about $10^{6}$ fissions, we claim that fission products alone will allow very high-confidence detection of atmospheric detonations, and that the major fission products may well cover up the minor fission products.

\section{Explanation of the Comments}

Table $3 \mathrm{a}$ and $3 \mathrm{~b}$ provided by Dr. Steve Lewis have been augmented in order to provide simple, isotope-by-isotope comments and are located at the bottom of this paper. The new columns are:

\begin{tabular}{ll}
\hline Nuclide Half Life & $\begin{array}{l}\text { A half life <ld means that after just a few days travel time, this isotope will be relatively } \\
\text { unimportant. }\end{array}$ \\
Nuclide Production Yield & $\begin{array}{l}\text { Especially low yields are reported in percentages. Anything low compared to } 6 \% \text { will not } \\
\text { have a good chance to be the first or second isotope. } \\
\text { Low gamma energy or low gamma abundance makes an isotope more vulnerable to } \\
\text { background. }\end{array}$ \\
A gamma ray in close proximity to a natural isotope or other fission product will either be \\
unusable or require special rehabilitation.
\end{tabular}

In addition, a column entitled 'Comments and Suggestions' reports a variety of problems or alternate selected gamma rays. For instance, certain isotopes are actually measured using daughter isotopes' gamma ray lines. Other isotopes are typically used as calibration standards and would be frequently detected as shine-through in the RASA and other CTBT RN aerosol systems. Table 4 is a direct comparison between the lists which comprise Table 3 and PrepCom Task Leader Paper TL 2/10.
Table 1. Low-yield Chinese Weapon Tests Detected in Richland, WA. RASA Fissions are computed by applying the

\begin{tabular}{cccc}
\hline Date & Putative Yield & Measured Fissions & RASA Fissions \\
\hline $10 / 16 / 64$ & $20 \mathrm{kt}$ & $7.54 \mathrm{E}+09$ & $4.43 \mathrm{E}+10$ \\
$5 / 14 / 65$ & $>20 \mathrm{kt}$ & $3.60 \mathrm{E}+09$ & $3.35 \mathrm{E}+09$ \\
$10 / 27 / 66$ & $<20 \mathrm{kt}$ & $7.67 \mathrm{E}+10$ & $7.14 \mathrm{E}+10$ \\
$12 / 24 / 67$ & $15-20 \mathrm{kt}$ & $1.10 \mathrm{E}+10$ & $1.02 \mathrm{E}+10$ \\
$11 / 18 / 71$ & $20 \mathrm{kt}$ & $1.47 \mathrm{E}+09$ & $2.45 \mathrm{E}+09$ \\
$1 / 7 / 72$ & $<20 \mathrm{kt}$ & $2.03 \mathrm{E}+09$ & $3.38 \mathrm{E}+09$ \\
$1 / 23 / 76$ & $<20 \mathrm{kt}$ & $2.57 \mathrm{E}+08$ & $4.28 \mathrm{E}+08$ \\
$9 / 17 / 77$ & $15 \mathrm{kt}$ & $6.05 \mathrm{E}+09$ & $1.01 \mathrm{E}+10$ \\
$3 / 15 / 78$ & $<20 \mathrm{kt}$ & $3.33 \mathrm{E}+10$ & $5.55 \mathrm{E}+10$ \\
$12 / 14 / 78$ & $<20 \mathrm{kt}$ & $3.30 \mathrm{E}+09$ & $5.50 \mathrm{E}+09$ \\
$10 / 16 / 80$ & $<20 \mathrm{kt}$ & $3.62 \mathrm{E}+08$ & $6.08 \mathrm{E}+08$ \\
\hline
\end{tabular}




\section{An Experiment}

In order to gain some idea of the effectiveness of various isotopes in detection, we created a fission product source by irradiating a uranium bearing liquid in a reactor. The liquid was calibrated for fission product content, and a small quantity was introduced as a source into a detector of very low background. The spectrum obtained from a 1 day count of the source at age 14 days was normalized to represent the result of the collection of $3.8 \times 10^{8}$ fissions ( $1 \mathrm{kt}$ explosion 10,000 $\mathrm{km}$ upwind). An automated analysis of the spectrum using the informal PNNL CTBT isotope library was performed. The resulting detected isotopes have been sorted in Table 2 .

Table 2. Sorted isotopes in a 14-day-old fission product spectrum. These isotopes are sorted by concentration and by error in the concentration (a measure of confidence). The library used did not contain all the isotopes in the DeGeer lists. Minimum Detectable Concentrations (MDC's) for unobserved isotopes are also shown in the Sorted by Concentration columns.

\begin{tabular}{|c|c|c|c|c|c|c|c|}
\hline \multicolumn{4}{|c|}{ Sorted by Error } & \multicolumn{4}{|c|}{ Sorted by Concentration } \\
\hline Rank & Isotope & Error & $\mathrm{Bq} / \mathrm{SCM}$ & Rank & Isotope & $\mathrm{Bq} / \mathrm{SCM}$ & Error \\
\hline 1 & Ce141 & 1.03 & $1.02 \mathrm{E}-02$ & 1 & Ba140 & $1.43 \mathrm{E}-02$ & 10.99 \\
\hline 2 & Te132 & 1.03 & $2.99 \mathrm{E}-03$ & 2 & La140 & 1.27E-02 & 9.01 \\
\hline 3 & Ru103 & 1.04 & $2.36 \mathrm{E}-03$ & 3 & $\mathrm{I} 132$ & $1.06 \mathrm{E}-02$ & 3.5 \\
\hline 4 & Ce144 & 1.94 & $1.75 \mathrm{E}-03$ & 4 & Ce141 & $1.02 \mathrm{E}-02$ & 1.03 \\
\hline 5 & $\mathrm{I132}$ & 3.5 & $1.06 \mathrm{E}-02$ & 5 & Nd147 & $4.24 \mathrm{E}-03$ & 23.23 \\
\hline 6 & Sb127 & 5.63 & $1.81 E-04$ & 6 & I131 & $3.47 \mathrm{E}-03$ & 9.59 \\
\hline 7 & Mo 99 & 6.84 & $1.47 \mathrm{E}-03$ & 7 & Te132 & $2.99 \mathrm{E}-03$ & 1.03 \\
\hline 8 & $\mathrm{Zr} 95$ & 8.66 & $2.16 \mathrm{E}-03$ & 8 & $\mathrm{Ru} 103$ & $2.36 \mathrm{E}-03$ & 1.04 \\
\hline 9 & Lal40 & 9.01 & $1.27 \mathrm{E}-02$ & 9 & $\operatorname{Zr} 95$ & $2.16 \mathrm{E}-03$ & 8.66 \\
\hline 10 & I131 & 9.59 & $3.47 \mathrm{E}-03$ & 10 & Ce144 & $1.75 \mathrm{E}-03$ & 1.94 \\
\hline 11 & Ba140 & 10.99 & $1.43 \mathrm{E}-02$ & 11. & Mo 99 & $1.47 \mathrm{E}-03$ & 6.84 \\
\hline 12 & Cs137 & 13.79 & $2.04 \mathrm{E}-05$ & 12 & Sb127 & $1.81 \mathrm{E}-04$ & 5.63 \\
\hline 13 & Cs136 & 15.06 & $2.65 \mathrm{E}-05$ & 13 & Cs136 & $2.65 \mathrm{E}-05$ & 15.06 \\
\hline 14 & Co 60 & $18.07^{\circ}$ & $1.53 \mathrm{E}-05$ & 14 & Cs137 & $2.04 \mathrm{E}-05$ & 13.79 \\
\hline \multirow[t]{12}{*}{15} & Nd147 & 23.23 & $4.24 \mathrm{E}-03$ & 15 & Co 60 & $1.53 \mathrm{E}-05$ & 18.07 \\
\hline & & & & \multicolumn{4}{|c|}{ MDC's for unobserved isotopes in library } \\
\hline & & & & & Isotope & $\mathrm{Bq} / \mathrm{SCM}$ & Error \\
\hline & & & & N/A & Ce143 & $1.89 \mathrm{E}-04$ & 100 \\
\hline & & & & $\mathrm{N} / \mathrm{A}$ & Ag111 & $1.52 \mathrm{E}-04$ & 100 \\
\hline & & & & N/A & 1133 & $1.05 \mathrm{E}-04$ & 100 \\
\hline & & & & N/A & K 40 & $6.09 \mathrm{E}-05$ & 100 \\
\hline & & & & N/A & $\operatorname{Zr} 97$ & $5.41 \mathrm{E}-05$ & 100 \\
\hline & & & & N/A & $\mathrm{Cd} 115$ & $3.76 \mathrm{E}-05$ & 100 \\
\hline & & & & N/A & $\operatorname{Ag} 110$ & $1.18 \mathrm{E}-05$ & 100 \\
\hline & & & & N/A & Cs134 & $1.03 \mathrm{E}-05$ & 100 \\
\hline & & & & N/A & $\mathrm{Na} 24$ & $1.73 \mathrm{E}-06$ & 100 \\
\hline
\end{tabular}

Note: The computation of error includes both statistical (counting) errors and other sources of error.

\section{Conclusions}

Our conclusion is that in a near worst case scenario where the plume avoids detection for 2 weeks and only one station intercepts, the signal should still be incontrovertible. A list of relevant nuclides for the purpose of screening events could be very short with little fear of missing any real event. Our recommendation is to eliminate activation products, consider elimination of certain problem fission products, and resist adding new fission products except for good reasons. In addition, we recommend that the gamma ray energies should either be deleted or at least have the energies rounded to the nearest $\mathrm{keV}$. Finally, we recommend the adoption of a single nuclear data source for halflives, gamma-ray energies, and branching ratios. 
Table 3a. Proposed Radionuclide List: Fission products.

\begin{tabular}{|c|c|c|c|c|c|c|c|c|c|}
\hline $\begin{array}{l}\text { Fission } \\
\text { Product } \\
\text { Nuclide }\end{array}$ & Half-life & & $\begin{array}{c}\text { Primary } \gamma \\
\text { energy } \\
\text { in } \mathrm{keV}^{*}\end{array}$ & $\begin{array}{c}\text { Primary } \gamma \\
\text { intensity } \\
\text { in } \%\end{array}$ & \begin{tabular}{|c|} 
Nuclide \\
Half Life
\end{tabular} & $\begin{array}{l}\text { Nuclide } \\
\text { Prod'n } \\
\text { Yield }\end{array}$ & \begin{tabular}{|c|} 
Nuclide \\
$\gamma$ Energy \\
or Abund
\end{tabular} & $\begin{array}{c}\gamma \\
\text { Interference }\end{array}$ & $\begin{array}{l}\text { Comments \& } \\
\text { Suggestions }\end{array}$ \\
\hline Strontium-91 & 9.63 & $\mathrm{~h}$ & 1024.3 & 33.4 & 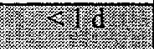 & & & & Also $556 \mathrm{keV}(61 \%)$ \\
\hline Yttrium-91 • & 58.51 & d & 1204.8 & 0.30 & & & 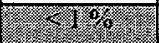 & & \\
\hline Yttrium-93 & 10.18 & $\mathrm{~h}$ & 266.9 & 7.32 & Tres: & & & & \\
\hline Zirconium-95 • & 64.02 & d & 756.7 & 54.5 & & & & & \\
\hline Niobium-95 • & 34.975 & d & 765.8 & 99.8 & & & & $768(\mathrm{Bi}-214)$ & \\
\hline Zirconium-97 • & 16.91 & $\mathrm{~h}$ & 743.4 & 93.1 & $\sqrt{2}$ & & & $\mathrm{Sb}-128$ & \\
\hline Molybdenum-99 • & 65.94 & $\mathrm{~h}$ & $140.5 \mathrm{D}$ & 89.4 & & & & Tc-99m & \\
\hline Technetium- $99 \mathrm{~m}$ & 6.01 & $\mathrm{~h}$ & 140.5 & 89.1 & & & & Mo-99 & \\
\hline Ruthenium-103 • & 39.26 & d & 497.1 & 90.9 & & & & & \\
\hline Rhodium-105• & 35.36 & $\mathrm{~h}$ & 319.1 & 19.2 & & & & & \\
\hline Ruthenium-106 & 373.59 & & $6219 \mathrm{D}$ & 9.93 & & & & & $\gamma$ is from Rh-106 daughter \\
\hline Palladium-109 & 13.712 & $\mathrm{~h}$ & 88.0 & 3.61 & & & Low E & & \\
\hline Silver-111 • & 7.45 & d & 342.1 & 6.7 & & & & & \\
\hline Palladium-112 & 21.03 & $\mathrm{~h}$ & $617.5 \mathrm{D}$ & 43 & & 00.0179 & & & \\
\hline Cadmium-115m & 44.6 & d & 933.8 & 2.0 & & 20.017 & 20. & $934(\mathrm{Bi}-214)$ & \\
\hline Cadmium-115 • & 53.46 & $\mathrm{~h}$ & 336.2 & 45.9 & & 80.017 & & $339\left(4 e^{2} 228\right)$ & \\
\hline Tin-123 & 129.2 & d & 1088.6 & 0.6 & & 20.077 & 6. & & \\
\hline Tin-125 • & 9.64 & d & 1067.1 & 10 & & & & & \\
\hline Antimony-125 • & 2.7582 & $\mathrm{y}$ & 427.9 & 29.6 & & & & & \\
\hline Antimony-126• & 12.46 & d & 695.0 & 99.6 & & & & Te-129m & Also $666 \mathrm{keV}(99.6 \%)$ \\
\hline Antimony-127 • & 3.85 & d & 685.7 & 36.8 & & & & & \\
\hline Tellurium-127m & 109 & d & 57.6 & 0.50 & & & 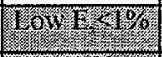 & & \\
\hline Tellurium-127 & 9.35 & $\mathrm{~h}$ & 418.0 & 0.99 & di & & 8 & 415 & \\
\hline Antimony-128 & 9.01 & $\mathrm{~h}$ & 743.2 & 100 & 31. & & & $\mathrm{Zr}-97$ & Also $754 \mathrm{keV}(100 \%)$ \\
\hline Tellurium-129m $\bullet$ & 33.6 & d & 695.9 & 3.19 & & & 175\% & Sb-126 & \\
\hline Iodine-130 & 12.36 & $\mathrm{~h}$ & 536.1 & 99 & & & & & $\begin{array}{l}\text { Also } 668 \mathrm{keV}(97 \%) \\
\text { Protected isotope }\end{array}$ \\
\hline Tellurium-131m & 30 & $\mathrm{~h}$ & 773.7 & 49.9 & & & & $\mathrm{Te}-132$ & \\
\hline Iodine-131 & 8.02070 & d & 364.5 & 81.7 & & & & & \\
\hline Tellurium-132 • & 3.204 & d & $772.6 \mathrm{D}$ & 75.6 & & & & Te-131m & Also $668 \mathrm{keV}(99 \%)$ \\
\hline Iodine-133 & 20.8 & $\mathrm{~h}$ & 529.9 & 87 & & & & Nd-147 & \\
\hline Iodine-135 & 6.57 & $\mathrm{~h}$ & 1260.4 & 28.9 & & & & & Also $1132 \mathrm{keV}(23 \%)$ \\
\hline Cesium-136• & 13.16 & d & 1048.1 & 80 & & & & & $\begin{array}{c}\text { Also } 818 \mathrm{keV}(100 \%) \\
\text { Protected isotope }\end{array}$ \\
\hline Cesium-137• & 30.07 & $\mathrm{y}$ & $661.7 \mathrm{D}$ & 85.1 & & & & & \\
\hline Barium-140 & 12.752 & d & 537.3 & 24.39 & & & & & \\
\hline Lanthanum-140 & 1.6781 & d & 1596.2 & 95.4 & & & & & \\
\hline Cerium-141 $\bullet$ & 32.501 & d & 145.4 & 48.2 & & & & & \\
\hline Cerium-143 • & 33.039 & $\mathrm{~h}$ & 293.3 & 42.8 & & & & $295(p 6-214)$ & \\
\hline Cerium-144 • & 284.893 & d & 133.5 & 11.09 & & & & & \\
\hline Neodymium-147 • & 10.98 & d & 531.0 & 13.1 & & & & $\mathrm{I}-133$ & \\
\hline Promethium-149 & 53.08 & $\mathrm{~h}$ & 286.0 & 3.1 & & & yes\% & $288(\mathrm{Br}-212)$ & \\
\hline Promethium-151 & 28.40 & $\mathrm{~h}$ & 340.1 & 22.5 & & & & $330(\mathrm{Ac}-228)$ & \\
\hline Samarium-153 & 46.27 & $\mathrm{~h}$ & 103.2 & 31.4 & & & & & \\
\hline Europium-155 • & 4.7611 & $\mathrm{y}$ & 105.3 & 21.2 & & & & & \\
\hline Samarium-156 & 9.4 & $\mathrm{~h}$ & 203.8 & 20.8 & & 50017 & & & \\
\hline Europium-156 • & 15.19 & d & 1153.7 & 6.8 & & & & $1155(\mathrm{Bi}-214)$ & Also $1231 \mathrm{keV}(89 \%)$ \\
\hline Europium-157 & 15.18 & $\mathrm{~h}$ & 370.5 & 11 & & 0.009 & & & Also $413 \mathrm{keV}(19 \%)$ \\
\hline
\end{tabular}


Table 3b Proposed Radionuclide List: Activation products.

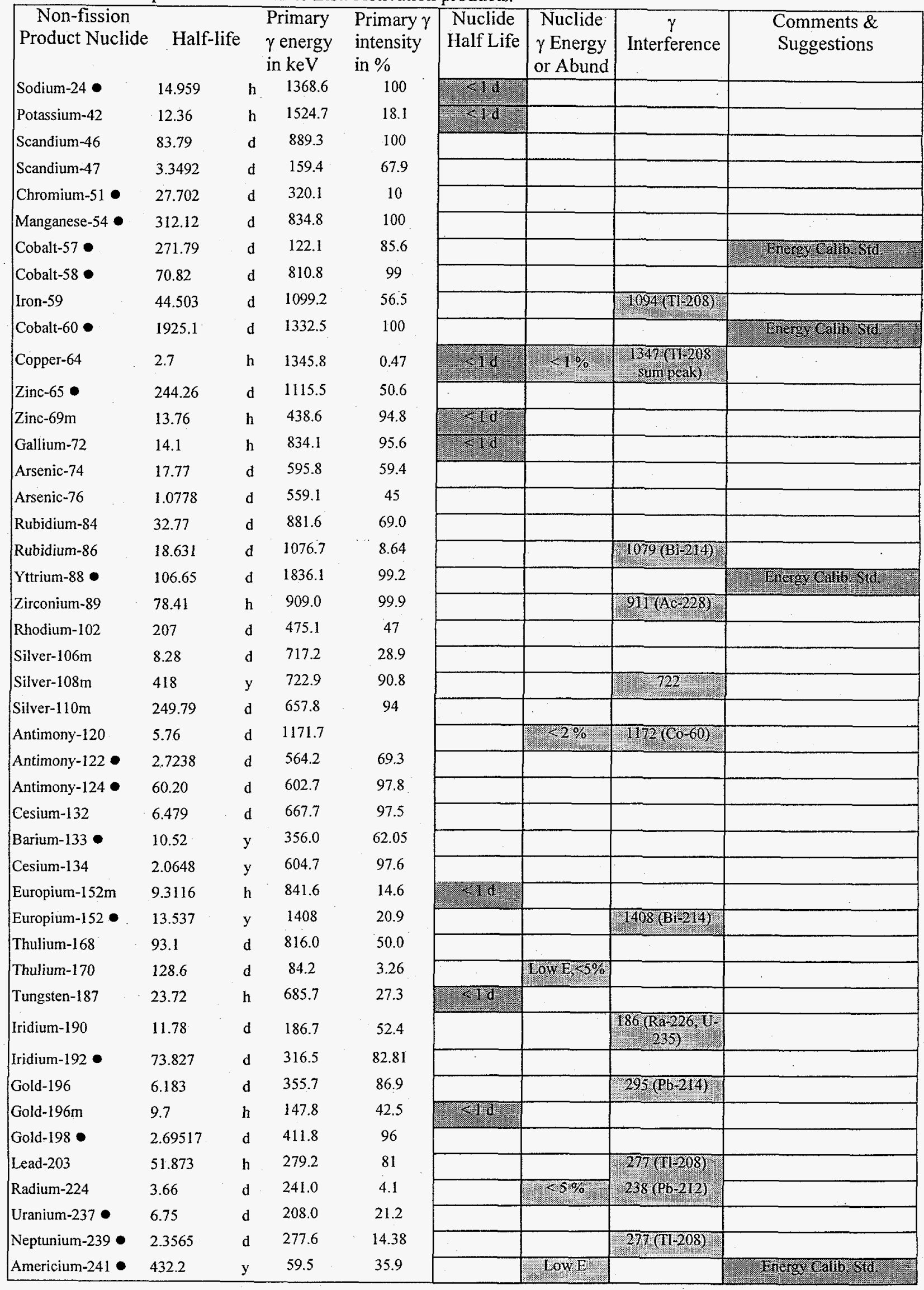




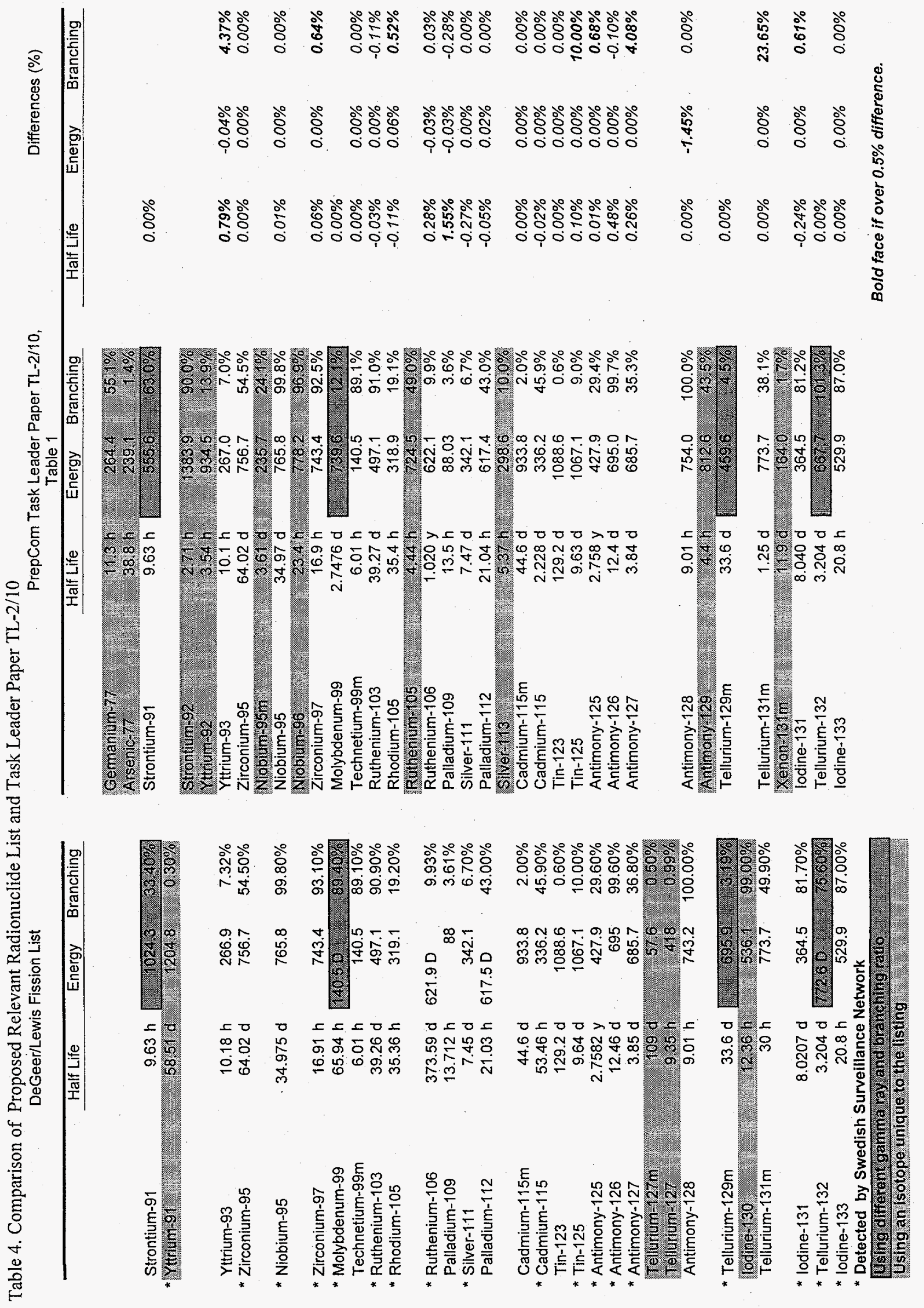




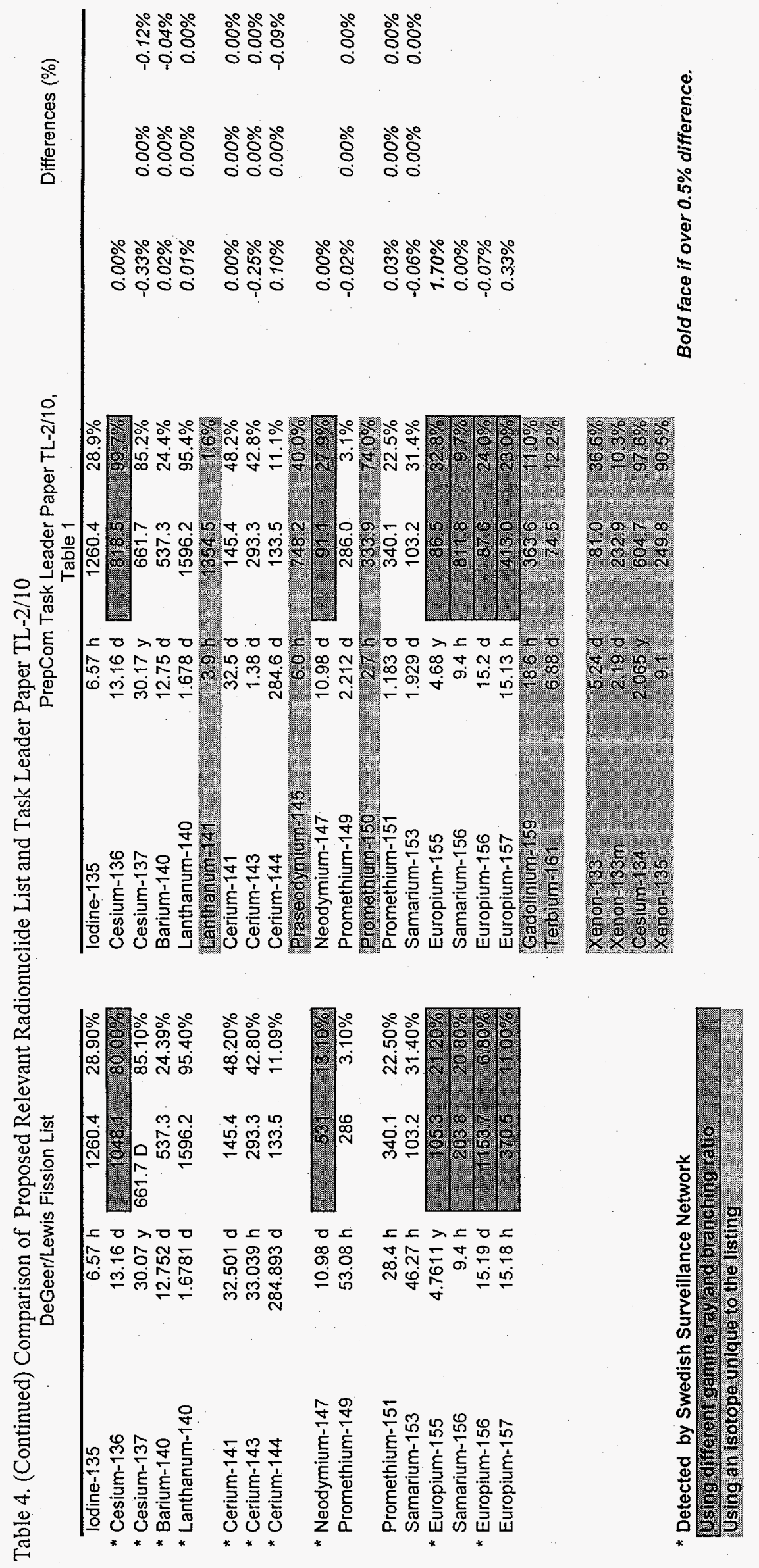

